\title{
COMUNICAÇÃO
}

\section{AVALIAÇÃO DE RESÍDUOS DE PAINÉIS DE MADEIRA GERADOS POR INDÚSTRIẢS MOVELEIRAS PARA APROVEITAMENTO NA CONFECÇÃO DE PEQUENOS OBJETOS - ESTUDO DE CASO}

\author{
Evaluation of residues of wood boards generated by furniture industries for their utilization in \\ the production of small objects - study of case
}

\author{
Luciana Barbosa de Abreu ${ }^{1}$, Lourival Marin Mendes², José Reinaldo Moreira da Silva², \\ Luana Elis de Ramos e Paula ${ }^{3}$, Fábio Akira Mori²
}

\begin{abstract}
RESUMO
A indústria moveleira produz uma quantidade de resíduos que, normalmente, é descartada no meio ambiente ou utilizada como combustível em caldeiras e similares, ou apenas queimada. Alternativas para utilização desses resíduos têm sido alvo de pesquisas, destacando-se a confecção de pequenos objetos, na forma de artesanato. Neste trabalho, objetivou-se avaliar os resíduos de indústrias do pólo moveleiro de Ubá/MG. No levantamento quantitativo, constatou-se que as empresas geraram resíduos dos mesmos tipos de painéis de madeira. Os resíduos mais comuns foram de aglomerado e MDF (Medium Density Fiber). Observou-se grande variabilidade das dimensões desses resíduos, principalmente em sua largura. Concluiu-se que alguns resíduos não apresentaram dimensões adequadas para a confecção de determinados objetos e que se torna necessária uma seleção prévia desses resíduos para direcioná-los a trabalhos mais minuciosos, de forma a viabilizar a sua utilização.
\end{abstract}

Termos para indexação: Resíduo de madeira, aproveitamento de resíduo, artesanato, indústria moveleira.

\begin{abstract}
The furniture industry generates an amount of residues, which, normally, is discarded in the environment or destined to steam production in boilers. The handmade production of small wood board objects is an outstanding alternative to take advantage of these residues. The general aim of this study was to evaluate residues in furniture industries located at the pole of Ubá/MG. Through the survey and the analysis of the data, one established that the industries generate residues from the same kind of wood board. The most common residues were of particleboard and MDF (Medium Density Fiber). One observed a great diversity of residue dimensions, mainly in width. One concluded that some residues did not present adequate dimensions for the manufacturing of some objects and that there is a necessity of previous residues selection, in order to destine them to more detailed works and make possible their use.
\end{abstract}

Index terms: Wood residue, utilization of residue, handmade art, furniture Industry.

(Recebido em 5 de setembro de 2006 e aprovado em 4 de julho de 2008)

No Brasil, a produção e a utilização de painéis de madeira reconstituída aumentaram muito nas últimas décadas. De acordo com Neves (1998), 60\% da utilização final dos painéis de partículas produzidos na América Latina são destinados às indústrias moveleiras. No entanto, o aproveitamento dos painéis não é integral. De 10 a $15 \%$ dos painéis de madeira reconstituída tornam-se resíduos em formas de aparas ou recortes (FERREIRA, 2003). Muitas indústrias moveleiras encaram seus resíduos como lixo proveniente do processo de produção e encontram problemas para sua destinação. Geralmente, os resíduos são acumulados, até que sejam enviados para caldeiras de geração de vapor e energia ou são depositados em áreas inapropriadas. Esse descarte tem causado poluição e sérios problemas ambientais, já que os painéis de madeira utilizam resinas sintéticas em sua composição.

Uma das maneiras de minimizar tais problemas é a reutilização de resíduos que podem ser aproveitados pela própria indústria ou vendidos para outras empresas para serem aplicados em usos de maior valor agregado. Se os resíduos são encarados como matéria-prima alternativa, eles deixam de ser um problema e passam a ser um

\footnotetext{
'Arquiteta e Urbanista, Doutoranda em Ciência e Tecnologia da Madeira - Departamento de Ciências Florestais/DCF - Universidade Federal de Lavras/ UFLA - Cx. P. 3037 - 37200-000 - Lavras, MG - luarquiteta@uol.com.br

${ }^{2}$ Engenheiros Florestais, Doutores em Ciências Florestais - Departamento de Ciências Florestais/DCF - Universidade Federal de Lavras/UFLA - Cx. P. 3037 - 37200-000 - Lavras, MG - lourival@ufla.br; jreinaldo@ufla.br; morif@ufla.br

${ }^{3}$ Engenheira Florestal, Mestranda em Ciência e Tecnologia da Madeira - Departamento de Ciências Florestais/DCF - Universidade Federal de Lavras/ UFLA - Cx. P. 3037 - 37200-000 - Lavras, MG - luanafloresta@hotmail.com
} 
subproduto da empresa em questão, podendo até gerar lucro (LIMA \& SILVA, 2005). Os resíduos se tornam uma opção interessante, dos pontos de vista econômico, ambiental e social. O seu aproveitamento pode proporcionar rendimento extra. Além disso, os impactos ambientais, criados quando os resíduos são depositados em locais inapropriados, não são desencadeados quando eles são aproveitados. A esses fatos, soma-se a criação de novas fontes de renda e de empregos para a comunidade. Se os resíduos de painéis de madeira são direcionados à confecção de pequenos objetos artesanais, agrega-se valor a esses resíduos.

Poucos são os estudos que apresentam alternativas técnica e economicamente eficazes para o aproveitamento de resíduos provenientes do processamento de painéis de madeira nas indústrias moveleiras.

A indústria de móveis em Minas Gerais está concentrada nas microrregiões de Belo Horizonte, Divinópolis e Ubá, que representam $44,9 \%$ do total de estabelecimentos do setor no estado. O principal pólo moveleiro do estado está localizado na Zona da Mata mineira, num território de nove municípios (Ubá, Guidoval, Rodeiro, Visconde do Rio Branco, São Geraldo, Guiricema, Tocantins, Piraúba e Rio Pomba) e conta com, aproximadamente, 350 indústrias (INSTITUTO EUVALDO LODI, 2003).

O diagnóstico em apenas uma empresa de um pólo moveleiro pode ser simples, conforme afirma Instituto Euvaldo Lodi (2003). Entretanto, quando se trata de uma gama de indústrias com características diversificadas e estruturas diferenciadas, todo tipo de ação se torna mais complexa. O diagnóstico sobre os resíduos de um pólo moveleiro tampouco é tarefa fácil. As características dos resíduos gerados em uma indústria variam muito, pois, dependem dos tipos de móveis que estão sendo feitos naquele período. Segundo Silva (1999), um fator que afeta quase a totalidade das indústrias é a flutuação da produção em razão da variação do volume comercializado ao longo do ano. Assim, os resultados obtidos sobre as características dos resíduos de alguma empresa em um determinado período de tempo não podem ser considerados representativos de sua produção anual e, portanto, não podem ser extrapolados para um grupo de empresas.

Um aspecto que também deve ser considerado é a falta de predisposição dos empresários em participar de estudos direcionados sobre a geração de resíduos em suas indústrias.

Diante da predisposição de três indústrias do pólo moveleiro de Ubá em participar de um estudo de caso, conduziu-se este trabalho, com o objetivo geral de coletar e avaliar os resíduos gerados durante um intervalo de duas horas de produção, em um dia normal de trabalho, com o intuito de destiná-los à produção de pequenos objetos artesanais. Especificamente, este trabalho buscou quantificar o volume gerado de cada painel processado por indústria e o volume total de resíduos gerado por indústria.

Dentro do universo de empresas do Pólo Moveleiro de Ubá, foram selecionadas, por meio de contatos com o Sindicato Intermunicipal das Indústrias de Marcenaria de Ubá, Intersind, três indústrias de médio porte dispostas a participar deste trabalho. As indústrias deveriam permitir a coleta de resíduos de sua produção, a fim de que eles fossem avaliados e, posteriormente, destinados à produção de objetos artesanais.

Para a coleta e quantificação dos resíduos de painéis de madeira foi deslocado um grupo de alunos da Universidade Federal de Lavras, UFLA, para a cidade de Ubá. Em cada uma das três indústrias foram feitos levantamentos dos resíduos gerados durante um período de duas horas de um ciclo normal de produção, durante o mês de junho de 2005. Para isso, foram utilizados paquímetros, réguas, trenas, além de planilhas onde foram anotados os dados levantados. Os dados consideraram a classificação quanto ao tipo de painel, o número de peças residuais, suas dimensões, bem como o volume total de resíduos gerados. Em seguida, foi feita uma avaliação quantitativa dos dados para cada empresa e para todas elas em geral.

A Tabela 1 apresenta o volume de resíduos de cada material gerado por cada empresa, em um período de duas horas de trabalho. Observou-se que a empresa A gerou $87,2 \%$ do total de seus resíduos de aglomerado; que a empresa B gerou $58,1 \%$ de MDF e que $86,1 \%$ dos resíduos gerados pela empresa $\mathrm{C}$ foram de $\mathrm{MDF}$.

A Figura 1 apresenta o percentual do volume total de resíduos por empresa. Observa-se que a empresa A é a maior geradora de resíduos, apresentando $57 \%\left(0,2847 \mathrm{~m}^{3}\right)$ do total. A empresa B gera $27,5 \%\left(0,1378 \mathrm{~m}^{3}\right)$ e a empresa C, $15,5 \%\left(0,0775 \mathrm{~m}^{3}\right)$ do total quantificado. O total de resíduos gerados pelas três empresas foi de $0,5000 \mathrm{~m}^{3}$ em duas horas de produção. Se não houvesse flutuação da produção, ou seja, se ela fosse mantida constante, em 8 horas de funcionamento dessas indústrias a produção de resíduos seria de aproximadamente $2 \mathrm{~m}^{3}$, um volume considerável para três empresas de médio porte. Vale lembrar que, como mencionado anteriormente, os tipos de móveis produzidos em uma empresa variam em diferentes épocas do ano e, conseqüentemente, resíduos de períodos desiguais apresentam diferenças entre si. O volume de resíduos por empresa certamente seria reduzido se elas comprassem painéis pré-cortados adequados às necessidades de sua produção.

Na Figura 2, pode-se observar que os resíduos gerados pelas três empresas são de aglomerado, compensado, lâminas e MDF. Observa-se que os resíduos de aglomerado são gerados em maior quantidade, com 53,5\% do total, os de MDF aparecem na segunda posição, com $31,2 \%$, os resíduos de compensado representam $7,5 \%$ do volume total e as lâminas, 7,8\%. 
Esse resultado se deve ao fato de o aglomerado ser, segundo Silva \& Oliveira (2001), o painel mais consumido pelas indústrias de móveis no Brasil. Ainda de acordo com esses autores, o compensado, que era o segundo painel mais consumido pelas indústrias, vem rapidamente perdendo espaço para o MDF, o que explica o fato de o volume de resíduos de MDF ter sido o segundo em volume gerado.

Os dados da Tabela 2 referem-se às médias de comprimento e largura dos resíduos de cada tipo de material. Observa-se que os resíduos de aglomerado apresentaram, em média, $53,6 \mathrm{~cm}$ de comprimento por $3,3 \mathrm{~cm}$ de largura; os resíduos de compensado possuem, em média, $56,8 \mathrm{~cm}$ por $5,0 \mathrm{~cm}$ e os de MDF apresentaram $51,5 \mathrm{~cm}$ por $3,8 \mathrm{~cm}$, em média. Os comprimentos seriam adequados à confecção de pequenos objetos, porém as larguras seriam estreitas para a confecção de determinados objetos.

Conforme ainda se pode observar na Tabela 2, os coeficientes de variação foram altos, principalmente para a largura. Esse dado indica alta variabilidade quanto às dimensões dos resíduos, fato explicado pelas empresas não trabalharem com cortes pré-determinados. Ainda assim, resíduos curtos e/ou estreitos não deixam de ser viáveis para a produção de pequenos objetos, uma vez que podem ser aproveitados para a confecção de um objeto diminuto ou para alguma parte de um objeto. Porém, se eles não forem separados dos resíduos maiores, provavelmente não serão selecionados e utilizados. Sendo assim, deve-se realizar a seleção prévia dos resíduos por classes de dimensões e direcioná-los de acordo com as necessidades dos artesãos.

Para confeccionar objetos, artesãos geralmente selecionam resíduos com dimensões maiores do que as que realmente necessitam. Esses resíduos são mais atrativos, pois se adaptam com mais facilidade às dimensões de vários objetos, oferecendo menores dificuldades para sua confecção. Porém, se os artesãos se esforçassem para usar materiais de formatos apenas um pouco maiores do que os objetos a serem produzidos, eles aproveitariam mais os materiais e não gerariam novos resíduos, além de poderem reservar os maiores formatos para quando precisassem confeccionar objetos maiores.

Tabela 1 - Volume $\left(\mathrm{m}^{3}\right)$ e percentagem com relação ao total do volume de resíduos de cada material, gerado por empresa em um período de 2 horas de trabalho.

\begin{tabular}{lcrcrcr}
\hline \multirow{2}{*}{ Material } & \multicolumn{7}{c}{ Empresa } \\
\cline { 2 - 7 } & Volume & \multicolumn{1}{c}{ A } & Volume & $\%$ & Volume & $\%$ \\
\hline aglomerado & 0,2482 & 87,2 & 0,0192 & 13,9 & 0,0000 & 0,0 \\
compensado & 0,0179 & 6,3 & 0,0181 & 13,1 & 0,0016 & 2,0 \\
Lâminas & 0,0092 & 3,2 & 0,0205 & 14,8 & 0,0092 & 11,9 \\
MDF & 0,0094 & 3,3 & 0,0800 & 58,1 & 0,0667 & 86,1 \\
\hline Total & 0,2847 & 100,0 & 0,1378 & 100,0 & 0,0775 & 100,0 \\
\hline
\end{tabular}

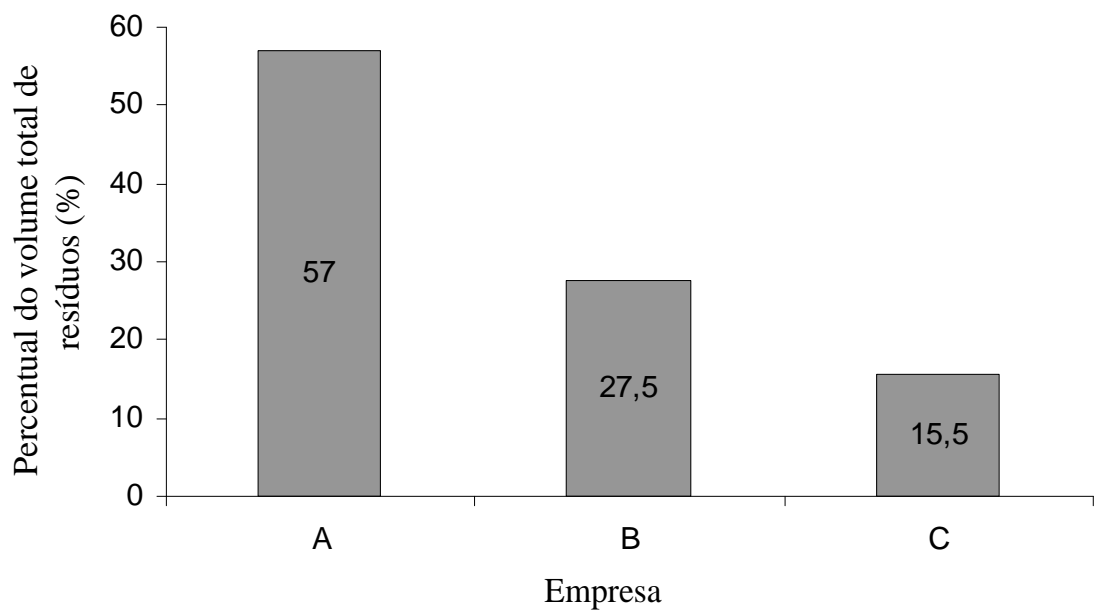

Figura 1 - Percentual do volume total de resíduos gerados pelas 3 empresas de Ubá, MG. 


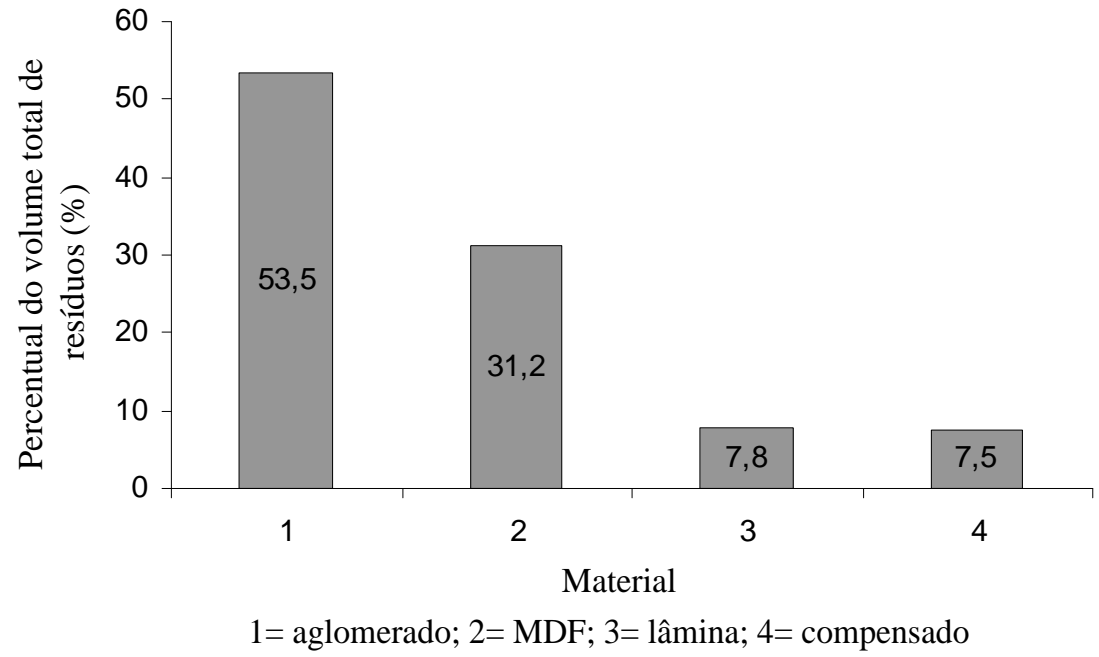

Figura 2 - Percentual do volume de resíduos de aglomerado, MDF, lâminas e compensado, gerados pelas três empresas de Ubá, MG.

Tabela 2 - Médias (cm) e coeficientes de variação (CV) de comprimento e de largura dos resíduos de cada material.

\begin{tabular}{lcccc}
\hline \multicolumn{1}{c}{ Material } & Comprimento & CV & Largura & CV \\
\hline aglomerado & 53,6 & $45,4 \%$ & 3,3 & $65,0 \%$ \\
compensado & 56,8 & $54,7 \%$ & 5,0 & $70,7 \%$ \\
MDF & 51,5 & $44,9 \%$ & 3,8 & $75,2 \%$ \\
\hline
\end{tabular}

No caso de pequenos objetos apresentarem alguns defeitos provenientes, por exemplo, da mistura de resíduos de painéis diferentes, não se deve perder de vista que se tratam de produtos artesanais, os quais, segundo Funarte (1980), são popularmente valorizados, mesmo se apresentarem pequenos defeitos. $\mathrm{O}$ fato de um artesanato ter certas irregularidades do padrão de fabricação apresenta, nesse aspecto, uma situação que pode não ser uma desvantagem, caso o artesão use de sua criatividade. Muitas vezes, o defeito vira ponto de interesse em um objeto. A irregularidade de um produto feito pela mão do homem é o toque de franca atração para o produtor artesanal (FUNARTE, 1980).

Concluiu-se, de acordo com as condições nas quais foi desenvolvido este estudo, que:

- os resíduos de painéis de madeira gerados pelas três empresas foram: aglomerado, compensado e MDF, além de lâminas decorativas;

- resíduos de aglomerado, com 54\% do total, e de MDF, com $31 \%$, foram os resíduos gerados em maior quantidade;
- houve uma grande variabilidade das dimensões dos resíduos, principalmente para a largura;

- alguns resíduos não apresentaram dimensões adequadas para utilização na confecção de determinados pequenos objetos;

- é necessária a seleção prévia de resíduos de menores dimensões, a fim de direcioná-los para trabalhos mais minuciosos e viabilizar a sua utilização.

\section{REFERÊNCIAS BIBLIOGRÁFICAS}

FERREIRA, S. Aproveitamento de resíduos de painéis de madeira para produção de artesanato. Lavras: UFLA, 2003. Projeto de pesquisa apresentado como parte das exigências da Disciplina DCF 537 - Industrialização da Madeira II - do Programa de Pós-graduação em Engenharia Florestal da Universidade Federal de Lavras.

FUNDAÇÃO NACIONAL DE ARTE. Artesanato brasileiro. 2. ed. Rio de Janeiro, 1980. 165 p.

INSTITUTO EUVALDO LODI-MG. Diagnóstico do pólo moveleiro de Ubá e região. Belo Horizonte: Intersind; Sebrae-MG, 2003. 90 p. 
LIMA, E. G.; SILVA, D. A. Resíduos gerados em indústrias de móveis de madeira situadas no pólo moveleiro de Arapongas-PR. Floresta, Curitiba, v. 35, n. 1, p. 203, jan./abr. 2005.

NEVES, M. R. Tendências dos mercados doméstico e internacional para produtos de base florestal. In: SEMINÁRIO DE ATUALIZAÇÃO. SISTEMAS DE COLHEITA DE MADEIRA E TRANSPORTE
FLORESTAL, 10., 1998, Curitiba, PR. Anais... Curitiba, 1998. p. 21.

SILVA, J. de C.; OLIVEIRA, J. T. da S. Diagnóstico do setor moveleiro no Brasil. Viçosa: UFV, 2001.

SILVA, J. R. M. Diagnóstico da indústria madeireira de Lavras/MG: relatório técnico: Fapemig. Lavras: UFLA, 1999. 